\title{
Phénoménologie de l’incarnation
}

\author{
Michel Henry
}

La chair, dès qu'elle n'est plus prise naïvement comme une donnée qui va de soi, dès que l'interrogation philosophique se lève sur son essence, i.e. sur la possibilité phénoménologique transcendantale qu'advienne dans notre expérience quelque chose comme une chair, renvoie à une présupposition incontournable: cette possibilité comme telle. La question en retour de la chair à ce qui la rend possible se laisse reconnaître dans l'histoire, à un moment crucial de celle-ci : lorsque le christianisme naissant se heurte de plein fouet aux intuitions de l'hellénisme. C'est alors en effet que la chair devient une préoccupation majeure dans la mesure où elle doit appartenir à Dieu aussi bien qu'à l'homme. Non pas à un Dieu quelconque - à ces dieux multiples, tous charnels, de l'Olympe, dont les aventures variées occupent agréablement l'imagination des hommes : c'est le Dieu invisible d'Abraham, cet absolu de justice qui entre en rapport avec lui et lui parle, en sorte que ce rapport intérieur à Dieu, vécu dans la foi, définit une existence nouvelle, celle d'Abraham et de toute sa descendance. C'est ce Dieu-là qui prend chair dans le christianisme, faisant de celle-ci un enjeu essentiel en même temps qu'un paradoxe déroutant.

Lors donc que le Verbe s'est fait chair et que celle-ci apparaît comme une propriété commune à Dieu et à l'homme, se pose la question de son origine - la question d'Apelles et de Tertullien : unde ?, d'où vient la chair ? D'où vient-elle de façon à pouvoir appartenir au Christ sans faire déchoir celui-ci de sa condition divine ? Cette possibilité a beau être définie de façon très particulière, elle n’en a pas moins une signification absolument générale, celle d’être la possibilité de toute chair, que ce soit celle du Christ ou la nôtre, puisqu'aussi bien la première est identique à la seconde.

Déplacée de son existence factuelle à son origine, i.e. à sa possibilité, la question de la chair est devenue celle de l'In-carnation. In-carnation désigne une venue dans la chair, en sorte que celle-ci renvoie à un Avant, qui est un Avant elle et à partir duquel elle advient. C'est cette venue qui est et doit être telle qu'à partir d'elle en effet, quelque chose comme de la chair soit possible. De manière inouïe, encore incomprise, Jean nomme cette origine, cet Avant-la-chair, cet Avant qui l'engendre proprement, faisant d'elle cette matière impressionnelle dont la phénoménologie de la chair a montré qu'elle est une matière phénoménologique pure. Ce en quoi vient toute chair, celle du Verbe notamment, ce qui de soi-même, par soi-même et en soi-même se fait chair, c'est le Verbe lui-même. 
Ainsi se trouvent en effet renversées, avant même qu'elles aient eu le temps de s'exprimer et de se développer, les positions de la gnose qui sont celles de l'hellénisme. Loin d'être un paradoxe insurmontable, la venue du Verbe dans une chair se dit comme la condition a priori de toute chair, se dit comme la condition a priori de toute chair concevable. L'Avant énigmatique de la chair, son origine vainement recherchée par la Gnose et objet de ses hypothèses imaginaires, est donc moins énigmatique que la chair elle-même. Comment en serait-il autrement ? Si la venue dans la chair précède celle-ci comme sa condition, alors cette venue est là, toujours déjà là quand une chair prend chair, quand à son tour elle est là. Seulement, elle n'est pas là, toujours déjà là, au sens d'un être-là dans le monde, au sens du Dasein. Être-là-dans-un-monde, c'est même ce que cette venue dans la chair n'est jamais. Pas plus que la chair d'ailleurs. C'est uniquement parce que, sur le plan d'une archi-facticité, la venue originaire dans une chair est étrangère au monde, qu'aucune chair factice n’y advient elle-même jamais ni ne se montre en lui.

Telle est maintenant l'évidence. Si ce qui vient avant toute chair et dont vient toute chair est le Verbe, alors en effet cette venue originaire est ce que nous savons le mieux. Car, ainsi qu'une phénoménologie radicale l'a reconnu à son premier pas, la venue originaire est celle de l'apparaître, c'est une venue de l'apparaître à l'apparaître dans l'apparaître lui-même. Quand cet auto-apparaître de l'apparaître a été élucidé en sa phénoménalité propre, celle-ci a été reconnue pour ce qu'elle est, cette auto-révélation de la révélation pure qui s'appelle la Vie. Comment cette auto-révélation de la Vie est son auto-génération, pour autant que venir à la vie veut dire venir dans la condition de s'éprouver soi-même - comment ce venir à la Vie génère en soi le Premier Soi en lequel la Vie s'étreint soi-même et ainsi se révèle à ce soi qui est son Verbe, comment ce Verbe de la Vie est aussi ancien qu'elle, pour autant que c'est en lui qu'elle vient en soi, c'est ce que la phénoménologie de la Vie a longuement montré. Comment ce Verbe maintenant porte en lui la possibilité de toute chair, se posant ainsi comme une Archi-Chair, cela résulte de ce que la venue de la Vie en son Verbe, qui est identiquement la venue du Verbe dans la Vie, est un Pathos : ce en quoi et ce pour quoi se phénoménalise la Vie, ce qui lui appartient dans le principe si le procès de sa venue en soi est celui de son auto-révélation. C'est ce Pathos impliqué en toute vie qui définit la possibilité transcendantale de toute chair concevable, l'Archi-Chair dont nous parlons.

Dans la mesure où elle tient sa possibilité et ainsi sa réalité d'une Archi-Chair qui la précède comme son origine et le lieu unique de son émergence, toute chair laisse paraître en elle la marque d'un retard. Or ce retard est d'une nature bien particulière, il n'indique aucun écart spatial ou temporel, aucune séparation, aucune Différence. C'est un retard sans retard, un retard qui ne se prive pas de la réalité pour la possession ou la jouissance de laquelle je me présente toujours en retard — mais qui me la donne. Je ne suis pas comme le voyageur qui se précipite à la gare pour n'apercevoir, en bout de quai, que la lanterne rouge du dernier wagon. Le train est 
toujours dans la station. C’est précisément parce qu'aucun écart ne me sépare de ce vis-à-vis de quoi je suis en retard à jamais que rien ne m'en sépare en effet, que toujours et déjà je suis en lui. Ce qui se trouve toujours déjà là pour moi dans le monde, dans mon intuition, dans ma pensée, c'est mon corps, mon corps mondain. Toujours déjà là pour moi comme autre que moi, incompréhensible à moi-même, obéissant à un destin étranger dont je lis avec stupeur les inéluctables développements sur les rides de mon visage, sur les derniers relevés d'analyses que me communique mon médecin. C'est de façon bien différente qu'une chair est toujours déjà là. Elle se trouve toujours déjà dans une chair, dans sa propre chair, en tant que chair. Sa chair n'est pas seulement ce qu'elle trouve, ce en quoi elle se trouve comme toujours déjà donnée impressionnellement à soi-même, c’est cette façon de se trouver soi-même comme toujours déjà donnée à soi dans sa propre chair, qui est toujours déjà là, cette façon toujours déjà à l'œuvre en laquelle toute chair est façonnée, donnée pathétiquement à soi comme cette chair s'éprouvant et s'impressionnant soi-même à la manière d'une chair. Mais ce n'est plus alors de celle-ci en sa facticité variable que nous parlons, c'est de cette "façon » - du Pathos de la Vie. C’est uniquement parce que, en son Archi-passibilité, la Vie vient en soi en s'éprouvant pathétiquement soi-même dans l'amour de son Verbe, en celui-ci donc, dans l'Archi-passibilité de son Archi-Chair, qu'une chair telle que la nôtre est possible. La phénoménologie de la chair est devenue une phénoménologie de l'Incarnation. La phénoménologie de l'Incarnation est une pièce essentielle de la phénoménologie de la Vie.

Dans sa référence à l'Avant de l'In-carnation, et parce que celle-ci renvoie à son tour à l'Archi-passibilité qui détermine la Vie en sa possibilité dernière, la chair manifeste une étrange affinité avec les autres déterminations essentielles de la condition humaine. Elle cesse de se présenter comme une addition contingente à celle-ci, une sorte d'appendice empirique, pour s'intégrer à un réseau de relations et de propriétés qui relève d'un a priori. Comment ne pas remarquer en effet que cette situation seconde de la chair par rapport à l'Archi-passibilité de la Vie est strictement parallèle à celle de l'ego comme du vivant en général ? Dans tous les cas, l'intelligibilité de ce qui est en question — le vivant, son ipséité, sa chair — implique que l'on se place en quelque sorte avant eux, dans une dimension d’origine. Celle-ci est précisément la même pour chacune des réalités considérées. À chacune elle retire la prétention qui est ordinairement la sienne d'être un principe ou un commencement, quelque entité autonome voire spécifique. Il y a un Avant-l'ego qui interdit à ce dernier de se poser comme un ultime fondement, ainsi qu'il est arrivé dans la philosophie moderne et encore dans la Krisis, lorsqu'elle reconnaît en lui «l'ego absolument unique fonctionnant en dernière instance » ${ }^{1}$.

\footnotetext{
${ }^{1}$ [Edmund Husserl, La crise des sciences européennes et la phénoménologie transcendantale, tr. fr. G. Granel, Paris, Gallimard (coll. « Tel »), 1999,] § 55, p. 212.
} 
Ce qui fonctionne n'est pas un ego. Avant la chair, là où elle est jointe à soi dans le Pathos de la Vie, il y a l'Archi-chair, l'Archi-passibilité dans laquelle aucune vie n'est concevable. Ainsi se découvre à nous la raison pour laquelle l'Avant-l'ego et l'Avant-la-chair ne font qu'un : c'est une même étreinte pathétique qui fait de la chair une chair et de l'ego un ego, l'auto-impressionnalité de la première et l'ipséité du second — l'auto-impressionnalité de la chair identique à l'ipséité de l'ego. Ainsi devient intelligible, archi-intelligible, l'appartenance principielle d'un ego à toute chair, d'une chair à tout ego. Ainsi s’affirme le progrès décisif accompli par la phénoménologie de l’In-carnation quand la corrélation ipséité/chair n’est plus déchiffrée sur une vie factice mais saisie à l'intérieur même du procès originaire de venue en soi de la Vie absolue. Car c'est de cette venue qu'il s'agit maintenant. C'est pourquoi l'Avant-l'ego et l'Avant-la-chair sont identiquement aussi un Avant-tout-vivant, en sorte que celui-ci, qui n'advient lui-même que dans ce procès de la Vie absolue, n'advient jamais que comme ce Soi charnel, ce Soi charnel vivant qui définit notre condition.

L’Évangile de Jean s'organise autour de deux Paroles fondatrices, qui vont déterminer le contenu dogmatique du christianisme. "Au début était le Verbe »; « Et le Verbe s'est fait chair ». La première se réfère au procès de la venue en soi de la Vie absolue, i.e. à la structure interne de Dieu. En elle se découvre l'originalité du monothéisme chrétien, celle de ne pas se réduire à l'affirmation formelle et conceptuelle d'un Dieu unique. Affirmation formelle dans la mesure où seule l'existence de Dieu est posée - un Dieu dont on ne sait rien, sinon qu'il existe. Parce qu'on ne sait rien de lui, son existence n'est précisément que l'objet d'une affirmation : suspendue à celle-ci, elle est aussi arbitraire qu'elle. Instruit par le Christ, Jean dit au contraire ce qu'est Dieu : il est Vie. La Vie ne peut demeurer à l'état de simple concept pour autant que vit un seul vivant, pour autant que moi je vis. Si je ne vivais pas d'ailleurs, il n'y aurait pour moi aucun concept de la vie.

Parce que moi qui vis je ne me suis pas apporté moi-même dans la vie, pas davantage dans ce Soi que je suis, non plus que dans ma chair, n’étant donné à moi-même qu'en elle — comme chair — alors ce vivant, ce Soi, cette chair ne viennent en eux que dans le procès de la Vie absolue qui vient en soi dans son Verbe — en lequel elle s'éprouve elle-même et s'aime elle-même, qui s'aime lui-même en elle, dans l'intériorité réciproque de leur amour qui est leur Esprit commun. C'est ainsi qu'à l'inverse du Dieu formel du monothéisme abstrait, le Dieu trinitaire chrétien est le seul Dieu réel, celui qui vit en chaque vivant, sans lequel aucun vivant ne vivrait, dont chaque vivant témoigne de par sa condition de vivant.

Le procès de la Vie en son auto-révélation pathétique dans le verbe, c'est donc là ce qu'énonce la première proposition de Jean. Parce que ce procès en lequel s'édifie une Archi-Chair porte en lui la possibilité de toute chair, la seconde proposition johannique est liée à la première. Toutes deux parlent du Verbe, la première en le rapportant à la Vie, la seconde à la chair. Maintenant, si toute chair vient de la Vie, 
alors la seconde proposition qui traite explicitement de la venue du Verbe dans une chair apparaît comme une conséquence de la première qui a non moins explicitement identifié le Verbe à la Vie, comme l'affirme le contexte immédiat: «En lui était la Vie ». Quelle sorte de «conséquence» est ici en jeu, c'est ce que la phénoménologie de l'In-carnation se propose d'établir en toute rigueur. D'ores et déjà cependant il apparaît que l'affrontement entre le christianisme primitif et la pensée grecque, le monde grec, est ici porté à son comble. Tandis que le logos grec — la pensée pure d'un intelligible irréductible au sensible, à la vie aussi bien qu'à la matière - se trouve dans le principe incapable de venir dans un corps - fût-il un corps vivant - le verbe chrétien, si rien ne le destine à prendre une chair comme la nôtre, n'en porte pas moins en lui, en tant que "Verbe de Vie", l'assise phénoménologique transcendantale de toute chair concevable. À l'impossibilité succède une possibilité, et une possibilité radicale. L'invraisemblable paradoxe chrétien n'est tel en fin de compte qu'au regard de la pensée grecque - au regard de la pensée. 



\section{LA PHENOMENOLOGIE FRANÇAISE AU JAPON}


